\title{
Powder Metallurgy of Non-Ferrous Metals: Part I
}

\author{
M. QIAN (1) ${ }^{1,2}$ \\ 1.-Centre for Additive Manufacturing, School of Engineering, RMIT University, Melbourne, \\ VIC 3000, Australia. 2.—e-mail: ma.qian@rmit.edu.au
}

At its meeting held on 28 February 2017 in San Diego, California, the TMS Powder Materials Committee decided to choose Powder Metallurgy of NonFerrous Metals as its technical emphasis for JOM in 2018. The proposed topic attracted 21 papers from the community. Included in this May issue of JOM are nine papers that were accepted following a rigorous peer review process. The key features of each accepted contribution are summarized as follows.

In the first article titled "Metal Injection Molding of Thin-walled Titanium Glasses Arms: A Case Study" by Ye et al., the authors reported an impressive development in the fabrication of long $(>200 \mathrm{~mm})$ and thin-walled titanium glasses arms with complex surface features using metal injection molding (MIM). Both commercially pure titanium and Ti-6Al-4V were demonstrated. The oxygen content in the as-sintered glasses arms is 0.22 wt.\% while the relative density is $>95 \%$. As a result, the as-sintered glasses frames exhibited good tensile mechanical properties.

The second article deals with "Experimental and Numerical Analysis of Injection Molding of Ti-6Al4V Powders for High Performance Titanium Parts." The authors showed that by using optimized processing conditions, MIM-processed Ti-6Al-4V samples can achieve excellent physical and mechanical properties (e.g., relative density: 99.8\%; ultimate tensile strength: $973 \mathrm{MPa}$; tensile elongation: $16 \%$ ).

In the third article titled "Advanced Mechanical Properties of a Powder Metallurgy Ti-Al-N Alloy Doped with Ultra-High Nitrogen," the authors presented an interesting study using nitrogen $(\mathrm{N})$ as an alloying element for the development of strong and ductile titanium alloys. As an interstitial impurity, $\mathrm{N}$ is tightly controlled in titanium materials. This study showed that extruded Ti-Al-N alloys

Ma Qian is the JOM advisor for the Powder Materials Committee of the TMS Materials Processing \& Manufacturing Division, and guest editor for the topic Powder Metallurgy of Non-Ferrous Metals: Part I in this issue. containing up to $0.81 \mathrm{wt} . \% \mathrm{~N}$ can exhibit excellent tensile properties superior to those of similarly extruded Ti-6Al-4V.

In the fourth article, Yang et al. report on "Producing High-Quality Titanium Alloy by a Cost-Effective Route Combining Fast Heating and Hot Processing." Two titanium alloys, Ti-5Al-5V$5 \mathrm{Mo}-3 \mathrm{Cr}$ ( $\mathrm{Ti}-5553$ ) and $\mathrm{Ti}-5 \mathrm{Fe}$, were fabricated from hydride-and-dehydride (HDH) titanium powder and master alloy powder using the proposed fast-heating and hot-processing route. High tensile strength was obtained with elongation in the range of $3 \%$ to $8 \%$, where both alloys contained high oxygen contents (0.39 wt.\% for Ti-5553 and 0.56 wt.\% for Ti$5 \mathrm{Fe}$ ).

The fifth article is concerned with "Selective Electron Beam Manufactured Ti-6Al-4V Strips: Effect of Build Orientation, Columnar Grain Orientation, and Hot Isostatic Pressing on Tensile Properties." Thin plate samples (3 mm thick) were built both horizontally and vertically, and their tensile mechanical properties were measured from long $(156 \mathrm{~mm})$ and thin $(2 \mathrm{~mm})$ samples before and after hot isostatic pressing (HIP). The vertically built strips showed clearly higher tensile strengths but lower elongation than the horizontally built ones. In particular, the authors were able to conclude that the retained prior- $\beta$ columnar grain boundaries after HIP showed no measurable influence on the tensile properties, irrespective of their length and orientation.

In the sixth article, Chen et al. presented a "Comparison Study on Additive Manufacturing (AM) and Powder Metallurgy (PM) AlSi10Mg Alloys." They showed that the selective laser melting (SLM) fabricated AlSi10Mg alloy exhibited coarser Al grains but much finer $\mathrm{Si}$ precipitates compared with the PM AlSi10Mg alloy. Consequently, they showed distinctly different tensile properties.

In the seventh article, the authors discussed in detail the "Mechanical Properties of Lightweight Porous Magnesium Processed through Powder 
Metallurgy." Four different porosity levels were produced, namely $28.4 \pm 1.8 \%, \quad 35.5 \pm 2.5 \%$, $45.4 \pm 1.9 \%$ and $62.4 \pm 2.2 \%$. Detailed compressive mechanical property data were produced and discussed. In addition, a comparison of mechanical properties was made between cancellous bone and porous magnesium produced by different space holders and processing methods.

The eighth article deals with "Synthesis of Tin Powder Using Tin Oxide and Ethanol." The authors reported an innovative method for the production of tin powder using tin oxide and ethanol and discussed the thermodynamics and kinetics of the proposed reduction process.

The ninth and final article in this collection is concerned with "Coercivity Enhancement of Nd-FeB HDDR Powders by Grain Boundary Diffusion Process with Rare-Earth Hydride" (HDDR: hydrogenation-disproportionation-desorption-recombination). The purpose is to realize the fabrication of dysprosium (Dy)-lean or Dy-free high-coercive NdFe-B permanent magnets because of the resource depletion issue of Dy. The authors used $\mathrm{NdHx}$ and $\mathrm{NdHx}-\mathrm{Cu}$ as diffusion sources for grain boundary modification of the HDDR powders and successfully enhanced the coercivity of the HDDR powders.

In summary, this selection of articles is published under the topic "Powder Metallurgy of Non-Ferrous Metals: Part I" in the May 2018 issue (vol. 70, no. 5) of $J O M$ and can be accessed via the JOM page at http://link.springer.com/journal/11837/70/5/page/1.

- "Metal Injection Molding of Thin-Walled Titanium Glasses Arms: A Case Study" by Shulong Ye, Wei Mo, Yonghu Lv, Xia Li, Chi Tat Kwok, and Peng Yu

- "Experimental and Numerical Analysis of Injection Molding of Ti-6Al-4V Powders for High
Performance Titanium Parts" by Dongguo Lin, Tae Gon Kang, Jun Sae Han, Seong Jin Park, Seong Taek Chung, and Young Sam Kwon

- "Advanced Mechanical Properties of a Powder Metallurgy Ti-Al-N Alloy Doped with Ultra-High Nitrogen" by J. Shen, B. Chen, J. Umeda, and K. Kondoh

- "Producing High-Quality Titanium Alloy by a Cost-Effective Route Combining Fast Heating and Hot Processing" by Fei Yang, Stella Raynova, Ajit Singh, Qinyang Zhao, Carlos Romero, and Leandro Bolzoni

- "Selective Electron Beam Manufactured Ti-6Al4V Strips: Effect of Build Orientation, Columnar Grain Orientation, and Hot Isostatic Pressing on Tensile Properties" by Jian Wang, Hui Ping Tang, Kun Yang, Nan Liu, Liang Jia, and Ma Qian

- "Comparison Study on Additive Manufacturing (AM) and Powder Metallurgy (PM) AlSi10Mg Alloys" by B. Chen, S.K. Moon, X. Yao, G. Bi, J. Shen, J. Umeda, and K. Kondoh

- "Mechanical Properties of Lightweight Porous Magnesium Processed through Powder Metallurgy" by Ning Zou and Qizhen Li

- "Synthesis of Tin Powder Using Tin Oxide and Ethanol" by Senol Cetinkaya and Serafettin Eroglu

- "Coercivity Enhancement of Nd-Fe-B HDDR Powders by Grain Boundary Diffusion Process with Rare-Earth Hydride" by Hee-Ryoung Cha, Jae-Gyeong Yoo, Youn-Kyoung Baek, DongHwan Kim, Hae-Woong Kwon, Yang-Do Kim, Dongyun Lee, and Jung-Goo Lee 